\title{
Applicability range of Stoney's formula and modified formulas for a film/substrate bilayer
}

\author{
Yin Zhang and Ya-pu Zhao \\ State Key Laboratory of Nonlinear Mechanics (LNM), Institute of Mechanics, Chinese Academy \\ of Sciences, Beijing 100080, People's Republic of China
}

(Received 28 April 2005; accepted 26 January 2006; published online 8 March 2006)

\begin{abstract}
In addition to the layer thickness and effective Young's modulus, the impact of the kinematic assumptions, interfacial condition, in-plane force, boundary conditions, and structure dimensions on the curvature of a film/substrate bilayer is examined. Different models for the analysis of the bilayer curvature are compared. It is demonstrated in our model that the assumption of a uniform curvature is valid only if there is no in-plane force. The effects of boundary conditions and structure dimensions, which are not (fully) included in previous models are shown to be significant. Three different approaches for deriving the curvature of a film/substrate bilayer are presented, compared, and analyzed. A more comprehensive study of the conditions regarding the applicability of Stoney's formula and modified formulas is presented. (C) 2006 American Institute of Physics.
\end{abstract}

[DOI: $10.1063 / 1.2178400]$

\section{INTRODUCTION}

Stoney's 1909 formula $^{1}$ serves as a cornerstone for the analysis of curvature based measurements. Stoney's formula has been modified many times for the purpose of evaluating bilayer and multilayer structures with arbitrary layer thickness ratios. ${ }^{2-13}$ Klein made comments on the accuracy of some modified Stoney formulas mentioned above. ${ }^{14}$ The assumption of a uniform curvature is explicitly or implicitly used in the context of bilayer or multilayer structures modeled as either beams or plates. ${ }^{2-13,15,16}$ Among these studies, Freund et al., in particular, presented an excellent analysis of the film/substrate bilayer and multilayer deflection due to lattice mismatch. ${ }^{7-9}$ Freund et al. also noted that the assumption of a uniform curvature is not valid for large nonlinear deformations. ${ }^{7,9}$ The validity of the kinematic assumption of a uniform curvature is examined in this paper. Two different models of the film/substrate system are examined and compared. It is demonstrated that the assumption of a uniform curvature is not valid even in the case of small linear deflections if there are in-plane forces. Usually the film/substrate longitudinal strain consists of two parts: the strain due to stretching and the strain due to bending. ${ }^{7}$ The stretching strain is directly related to the in-plane force, which can significantly affect the deflection. Physically, thermal and intrinsic stresses due to film growth are the two major sources of in-plane stresses and forces. ${ }^{15}$

A model that excludes the kinematic assumption of a uniform curvature is presented. The assumption of a uniform curvature leads to a conclusion that structural length and boundary conditions have no influence on the curvature. We demonstrate that this is true only if there is no in-plane force. The effect of the interface condition on the curvature is also discussed. Specifically, this paper presents an analysis of the deflection of an isotropic film/substrate bilayer due to lattice

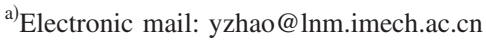

mismatch as a demonstration example. The analysis can be extended to the bilayer deflection induced by other mechanisms.

\section{LATTICE MISMATCH INDUCED DEFLECTION OF A FILM/SUBSTRATE BILAYER}

\section{A. Strain distribution due to lattice mismatch}

In Fig. $1, M_{f}$ and $h_{f}$ are the effective Young's modulus and layer thickness of the film. $M_{s}$ and $h_{s}$ are the effective Young's modulus and layer thickness of the substrate. For the heteroepitaxial growth of a film on a substrate, the mismatch strain $\epsilon_{m}$ is defined as ${ }^{7}$

$$
\epsilon_{m}=\frac{a_{s}-a_{f}}{a_{f}} .
$$

where $a_{f}$ and $a_{s}$ are the lattice parameters of the film and the substrate, respectively. The lattice-mismatch induced strain in the film $\epsilon_{f}$ and the lattice-mismatch induced strain in the substrate $\epsilon_{s}$ are determined by the following equations: ${ }^{7}$

$$
\begin{aligned}
& \epsilon_{f}-\epsilon_{s}=\epsilon_{m}, \\
& M_{f} \epsilon_{f} h_{f}+M_{s} \epsilon_{s} h_{s}=0 .
\end{aligned}
$$

The first equation is the compatibility condition. The second derives from Newton's third law, which states that the force acting in the film layer has the same magnitude but the opposite direction of that acting in the substrate. Here the film and substrate are assumed to have the same uniform width. The strains $\epsilon_{f}$ and $\boldsymbol{\epsilon}_{s}$ as obtained from Eq. (2) are as follows:

$$
\epsilon_{f}=\epsilon_{m} \frac{M_{s} h_{s}}{M_{f} h_{f}+M_{s} h_{s}}, \quad \epsilon_{s}=-\epsilon_{m} \frac{M_{f} h_{f}}{M_{f} h_{f}+M_{s} h_{s}} .
$$

Equation (3) clearly indicates that if the strain of one layer is negative, that of the other layer is positive. This implies that there is a bending moment due to the mismatch strain, and 
therefore, the film/substrate system will be subject to bending deformation.

\section{B. Circular film/substrate system}

Figure 1(a) specifies the cylindrical coordinate system. The film and substrate materials are assumed isotropic and the deformation is assumed axisymmetric. For the in-plane normal strains, $\epsilon_{r r}$ and $\epsilon_{\theta \theta}$, the following kinematic assumption holds: ${ }^{7}$

$$
\epsilon_{r r}=\epsilon_{\theta \theta}=\left\{\begin{array}{l}
\epsilon_{o}+\epsilon_{f}-\kappa z, \quad \frac{1}{2} h_{s}<z<\frac{1}{2} h_{s}+h_{f} \\
\epsilon_{o}+\epsilon_{s}-\kappa z, \quad-\frac{1}{2} h_{s}<z<\frac{1}{2} h_{s}
\end{array}\right.
$$

$\epsilon_{o}$ is the uniform in-plane strain due to stretching. The symbol $\kappa$ designates the curvature. The film/substrate system has the following potential energy:

$$
\begin{aligned}
V\left(\epsilon_{o}, \kappa\right)= & 2 \pi\left[\int_{0}^{R} \int_{1 / 2 h_{s}}^{1 / 2 h_{s}+h_{f}} M_{f}\left(\epsilon_{o}+\epsilon_{f}-\kappa z\right)^{2} r d z d r\right. \\
& \left.+\int_{0}^{R} \int_{-1 / 2 h_{s}}^{1 / 2 h_{s}} M_{s}\left(\epsilon_{o}+\epsilon_{s}-\kappa z\right)^{2} r d z d r\right],
\end{aligned}
$$

where $R$ is the radius of the film/substrate system. The equilibrium requires the potential energy $V\left(\epsilon_{o}, \kappa\right)$ to be stationary and therefore,

$$
\frac{\partial V\left(\epsilon_{o}, \kappa\right)}{\partial \epsilon_{o}}=0, \quad \frac{\partial V\left(\epsilon_{o}, \kappa\right)}{\partial \kappa}=0 .
$$

Note that no boundary conditions are required here to derive the curvature $\kappa$. From Eq. (6), $\kappa$ is found equal to $\kappa_{F}$, which is

$$
\kappa_{F}=\frac{\kappa_{\mathrm{St}}\left[1+\left(h_{f} / h_{s}\right)\right]}{1+4\left(h_{f} / h_{s}\right)\left(M_{f} / M_{s}\right)+6\left(h_{f} / h_{s}\right)^{2}\left(M_{f} / M_{s}\right)+4\left(h_{f} / h_{s}\right)^{3}\left(M_{f} / M_{s}\right)+\left(h_{f} / h_{s}\right)^{4}\left(M_{f} / M_{s}\right)^{2}} .
$$

$\kappa_{F}$ is the curvature as derived by Freund though Freund set $\epsilon_{f}=\epsilon_{m}$ and $\epsilon_{s}=0$ in Eq. (4). ${ }^{7}$ The symbol $\kappa_{\mathrm{St}}$ designates Stoney's curvature and is defined as follows: ${ }^{7}$

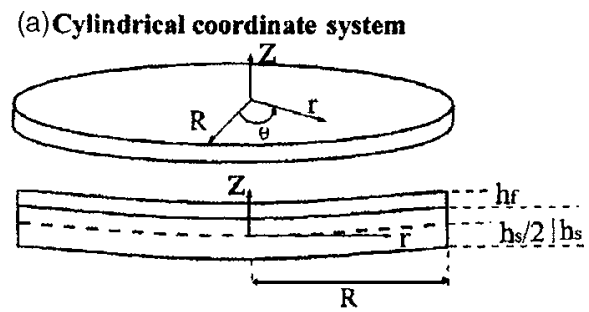

(b) Coordinate systems in Timoshenko's model

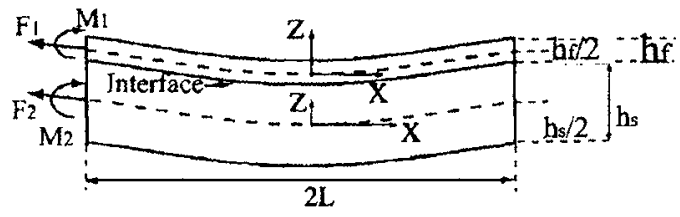

(c) Coordinate system in our method

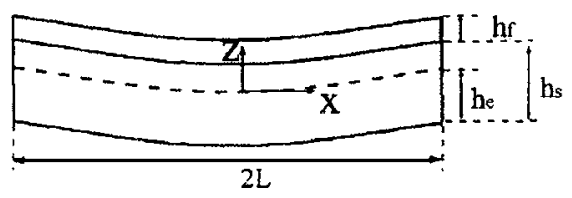

FIG. 1. (a) Schematic diagram of the film/substrate system in cylindrical coordinates. $R$ is the radius. The film has an effective Young's modulus $M_{f}$ and a thickness $h_{f}$. The substrate has an effective Young's modulus $M_{s}$ and a thickness $h_{s}$. (b) Rectangular coordinate systems as in Timoshenko's model. $M_{1}$ and $F_{1}$ are the moment and the force per unit width acting in the film layer. $M_{2}$ and $F_{2}$ are the moment and the force per unit width acting in the substrate layer. (c) shows the rectangular coordinate system in energy method. The abscissa originates at the neutral plane of the film/substrate composite structure.

$$
\kappa_{\mathrm{St}}=\frac{6 f}{M_{s} h_{s}^{2}}=\frac{6 M_{f} h_{f} \epsilon_{m}}{M_{s} h_{s}^{2}} .
$$

For $h_{f} / h_{s} \ll 1, f$ represents the surface stress and

$$
\lim _{h f / h_{s} \ll 1} \kappa_{F}=\kappa_{\mathrm{St}} .
$$

Note that $\epsilon_{o}$ and $R$ do not appear in the $\kappa_{F}$ expression as given by Eq. (7). In Eq. (6), an implicit assumption of a uniform curvature is made. ${ }^{7}$ We will demonstrate that if Eq. (4) applies, the assumption is true. However, in the next sections, we will demonstrate that a different and refined kinematic assumption rather than Eq. (4) should be used during the derivation. When the refined kinematic assumption applies, the assumption of a uniform curvature is not true any more when $\epsilon_{o} \neq 0$. Now returning to Eq. (4), we replace $\kappa$ by $v_{r r}$ and treat $\epsilon_{o}$ as a variable parameter. The symbol $v$ designates the transverse displacement and $v_{r r}=d^{2} v / d r^{2}$. By applying the principle of minimum potential energy (PMPE), which means $\delta V=0$, we derive the following governing equation

$$
\frac{d}{d r}\left[\frac{d}{d r}\left(r \frac{d^{2} v}{d r^{2}}\right)\right]=0
$$

We solve this equation and find the curvature by letting $\kappa$ $=v_{r r}$. The essential difference between Eqs. (10) and (6) is that there is no uniform curvature assumption in Eq. (10). Equation (10) is solved to obtain

$$
v(r)=C_{1} r^{2}+C_{2} r \ln r+C_{3} r+C_{4},
$$

where $C_{i}(i=1,2,3,4)$ are constants to be determined. Since the system is axisymmetric, $d v / d r=0$ at the center $(r=0)$, and 


$$
\frac{d v}{d r}=2 C_{1} r+C_{2}+C_{2} \ln r+C_{3}
$$

Because $\lim _{r \rightarrow 0}(\ln r) \rightarrow \infty, C_{2}=0$. Therefore, independent of the boundary conditions at $r=R$, the curvature $\left(v_{r r}\right)$ remains uniform $\left(v_{r r}=2 C_{1}\right)$. We also note that if Eq. (4) applies, $\epsilon_{o}$ does not appear in either the governing equation or the boundary conditions.

\section{Timoshenko's model}

Figure 1(b) shows the two rectangular coordinate systems of Timoshenko's model. Timoshenko's 1925 paper concerns with the thermal stress induced deflection of a bilayered composite made of two materials with different coefficients of thermal expansion. ${ }^{17}$ The essence of Timoshenko's model is to assume that an unknown force couple acts in the upper and lower layers. The unknown force couple is related to the curvature of the bilayer and determined by an interfacial constraint condition. Recently, Yang and Li extended this concept to the diffusion-induced deflection of bilayered and multilayered beams. ${ }^{16}$ Here we summarize Timoshenko's model and apply it to the lattice-mismatch induced deflection of a film/substrate bilayer.

In Fig. 1(b), $F_{1}$ and $F_{2}$ are the forces per unit width acting in the upper and lower layers; $M_{1}$ and $M_{2}$ are the moments per unit width acting in the upper and lower layers. The equilibrium requires the balance of both force and moment, therefore,

$$
F_{1}+F_{2}=0
$$

and

$$
M_{1}+M_{2}-F_{1} \frac{h_{f}}{2}+F_{2} \frac{h_{s}}{2}=0
$$

$F_{1}$ and $F_{2}$ are the force couple to be solved. From Eq. (13), we have

$$
F_{1}=P=-F_{2} \text {. }
$$

Substitute it into Eq. (14), we have

$$
\frac{P\left(h_{f}+h_{s}\right)}{2}=M_{1}+M_{2} \text {. }
$$

The in-plane (longitudinal) strains of the film and substrate, as in the beam theory, can be expressed as follows:

$$
\begin{gathered}
\frac{d u_{f}(x)}{d x}=\epsilon_{o}+\epsilon_{f}+\frac{P}{M_{f} h_{f}}-\frac{z}{\rho}, \quad-\frac{h_{f}}{2} \leqslant z \leqslant \frac{h_{f}}{2} \\
\frac{d u_{s}(x)}{d x}=\epsilon_{o}+\epsilon_{s}-\frac{P}{M_{s} h_{s}}-\frac{z}{\rho}, \quad-\frac{h_{s}}{2} \leqslant z \leqslant \frac{h_{s}}{2},
\end{gathered}
$$

where $u_{f}$ and $u_{s}$ are the in-plane displacements of the film and substrate, respectively. If $\epsilon_{o}+\epsilon_{f}$ and $\epsilon_{o}+\epsilon_{s}$ represent thermally induced strains of layers, Eq. (17) matches the original Timoshenko's model. For a beam structure, $M_{f}=E_{f}$ and $M_{s}$ $=E_{s}$. For a plate structure that bends to a cylindrical surface, $M_{f}=E_{f} /\left(1-\nu_{f}^{2}\right)$ and $M_{s}=E_{s} /\left(1-\nu_{s}^{2}\right)\left(E_{f}\right.$ and $E_{s}$ and $\nu_{f}$ and $\nu_{s}$ are Young's moduli and Poisson's ratios of the film and substrate, respectively). ${ }^{18}$ The symbol $\rho$ designates the radius of the curvature. In the coordinate systems shown in Fig. 1(b), $1 / \rho=d^{2} v_{f}(x) / d x^{2}=d^{2} v_{s}(x) / d x^{2}$, where $v_{f}(x)$ and $v_{s}(x)$ are the transverse deflections of the midplanes of the film and substrate, respectively. In general, $v_{f}(x) \neq v_{s}(x)$, but their derivatives are assumed to be the same. ${ }^{19}$ The linear curvaturemoment relation yields the following equations:

$$
M_{1}=-\frac{M_{f} h_{f}^{3}}{12 \rho}, \quad M_{2}=-\frac{M_{s} h_{s}^{3}}{12 \rho} .
$$

Substituting Eq. (18) into Eq. (16), we have

$$
\kappa_{T}=\frac{1}{\rho}=-\frac{6 P\left(h_{f}+h_{s}\right)}{M_{f} h_{f}^{3}+M_{s} h_{s}^{3}} .
$$

Equation (19) gives the curvature of Timoshenko's model. So far, $P$ is still unknown. To obtain $P$, the compatibility condition at the interface is used, i.e.,

$$
\epsilon_{o}+\epsilon_{f}+\frac{P}{M_{f} h_{f}}+\frac{h_{f}}{2 \rho}=\epsilon_{o}+\epsilon_{s}-\frac{P}{M_{s} h_{s}}-\frac{h_{s}}{2 \rho} .
$$

Physically, Eq. (20) states that there is no slip at the interface, ${ }^{20}$ which is an ideal case. In this paper, we only model and discuss the ideal case. However, in real world situations, interfacial slip occurs. For example, the formation of an amorphous layer and dangling bonds in some regions between the two crystals results in the weakly bonded interface areas. ${ }^{21-23}$ This will reduce the overall interface adhesion. ${ }^{23}$ For a nonideal or damaged interface, ${ }^{24}$ the interfacial slip arises. Both experiment and theoretical analyses show that the interfacial slip greatly influences the stress distribution inside the layer. ${ }^{20,25}$ Therefore, the deflection and curvature of the bilayer are affected. The interface layer models including the shear-lag model ${ }^{26}$ and lap-shear model $^{27,28}$ can be used to model the nonideal case by allowing interfacial slip. ${ }^{20}$ From Eq. (20), $P$ is found to be

$$
P=\frac{-\epsilon_{m}}{1 / M_{f} h_{f}+1 / M_{s} h_{s}+3\left(h_{f}+h_{s}\right)^{2} /\left(M_{f} h_{f}^{3}+M_{s} h_{s}^{3}\right)},
$$

keeping in mind that $\epsilon_{f}-\epsilon_{s}=\epsilon_{m}$. Again, we note that $\epsilon_{o}$ and length $L$ do not appear in the curvature solution in Timoshenko's model. The boundary conditions are not used, either. If $h_{f} / h_{s} \ll 1$, Eq. (21) reduces to

$$
\lim _{h f / h_{s} \ll 1} P=-M_{f} h_{f} \epsilon_{m}=-f,
$$

and Eq. (19) yields

$$
\lim _{h f / h_{s} \ll 1} \kappa_{T}=-\frac{6 P}{M_{s} h_{s}^{2}}=\frac{6 f}{M_{s} h_{s}^{2}}=\kappa_{\mathrm{St}} .
$$

Unlike Timoshenko's model, the derivation of $\kappa_{F}$ does not assume a constraint force couple. In Eq. (4), continuity of strain across the interface is assumed, and the effective Young's moduli of the two layers are different. This results in the discontinuity of stress across the interface, which is equivalent to the constraint force couple in Timoshenko's model. A different approach adopted by Röll, ${ }^{2}$ Klein and Miller, ${ }^{10}$ Hsueh, ${ }^{11}$ and $\mathrm{Hu}$ and Huang ${ }^{13}$ also leads to $\kappa_{T}$. They first postulate an equation similar to Eq. (4) and then use the 
equilibrium conditions of Eqs. (13) and (14) to derive the curvature.

For arbitrary $h_{f} / h_{s}$, no one has pointed out that the relation, ${ }^{29}$

$$
\kappa_{F}=\kappa_{T},
$$

holds.

\section{In-plane force and boundary condition effects}

Figure 1(c) shows the rectangular coordinate system for a film/substrate bilayer. In this coordinate system, as the ordinate originates at the neutral plane, Eq. (4) must be rewritten as follows:

$$
\epsilon_{x x}= \begin{cases}\epsilon_{o}+\epsilon_{f}-z \frac{d^{2} v(x)}{d x^{2}}, & h_{s}-h_{c}<z<h_{s}+h_{f}-h_{c} \\ \epsilon_{o}+\epsilon_{s}-z \frac{d^{2} v(x)}{d x^{2}}, & -h_{c}<z<h_{s}-h_{c},\end{cases}
$$

where $\epsilon_{x x}$ is the in-plane strain, and $v(x)$ is the deflection of the neutral plane, which is located $\mathrm{at}^{30}$

$$
h_{c}=\frac{M_{f} h_{f}^{2}+M_{s} h_{s}^{2}+2 M_{f} h_{f} h_{s}}{2 M_{f} h_{f}+2 M_{s} h_{s}} .
$$

If $h_{f} / h_{s} \ll 1, h_{c}=1 / 2 h_{s}$, i.e., for a very thick substrate, the neutral plane is the middle plane of the substrate. If a nonlinear term is added to Eq. (25), ${ }^{7}$ we have

$$
\epsilon_{x x}=\left\{\begin{array}{c}
\epsilon_{o}+\epsilon_{f}-z \frac{d^{2} v(x)}{d x^{2}}+\frac{1}{2}\left[\frac{d v(x)}{d x}\right]^{2}, \\
h_{s}-h_{c}<z<h_{s}+h_{f}-h_{c} \\
\epsilon_{o}+\epsilon_{s}-z \frac{d^{2} v(x)}{d x^{2}}+\frac{1}{2}\left[\frac{d v(x)}{d x}\right]^{2}, \\
-h_{c}<z<h_{s}-h_{c} .
\end{array}\right.
$$

As explained in Ref. 31, the nonlinear term $\frac{1}{2}[d v(x) / d x]^{2}$ due to neutral plane stretching will significantly affect the structure deflection and dynamics even for small deflections if $\epsilon_{o} \neq 0$. The potential energy of the bilayer now amounts to

$$
\begin{aligned}
V= & \frac{1}{2} \int_{-L}^{L}\left[\int_{h_{s}-h_{c}}^{h_{s}+h_{f}-h_{c}} M_{f}\left(\epsilon_{o}+\epsilon_{f}-z v_{x x}+\frac{v_{x}^{2}}{2}\right)^{2} d z\right. \\
& \left.+\int_{-L}^{L} \int_{-h_{c}}^{h_{s}-h_{c}} M_{s}\left(\epsilon_{o}+\epsilon_{f}-z v_{x x}+\frac{v_{x}^{2}}{2}\right)^{2} d z\right] d x,
\end{aligned}
$$

where $v_{x x}=d^{2} v / d x^{2}$ and $v_{x}=d v / d x$. Applying the PMPE $(\delta V=0)$, we derive both the governing equation and the boundary conditions for the bilayer. The following governing equation is obtained:

$$
K_{4} v_{x x x x}-K_{2} v_{x x}-6 K_{5} v_{x}^{2} v_{x x}=0 .
$$

Here we define $K_{i}(i=1-5)$ as follows:

$$
\begin{aligned}
K_{1} & =-M_{s} \epsilon_{s}\left(h_{s}^{2}-2 h_{s} h_{c}\right)-M_{f} \epsilon_{f}\left(h_{f}^{2}+2 h_{f} h_{s}-2 h_{f} h_{c}\right) \\
& =-M_{f} \epsilon_{f}\left(h_{f}^{2}+h_{f} h_{s}\right), \\
K_{2} & =\left(M_{s} h_{s}+M_{f} h_{f}\right) \epsilon_{o},
\end{aligned}
$$

$$
\begin{aligned}
& K_{3}=-\frac{\left(h_{s}^{2}-2 h_{s} h_{c}\right) M_{s}}{2}-\frac{\left(h_{f}^{2}+2 h_{f} h_{s}-2 h_{f} h_{c}\right) M_{f}}{2}, \\
& K_{4}=\frac{\left(h_{s}-h_{c}\right)^{3}+h_{c}^{3}}{3} M_{s}+\frac{\left(h_{f}+h_{s}-h_{c}\right)^{3}-\left(h_{s}-h_{c}\right)^{3}}{3} M_{f},
\end{aligned}
$$

$$
K_{5}=\frac{M_{f} h_{f}+M_{s} h_{s}}{4} .
$$

This derivation makes use of Eqs. (2) and (26). Note that $-\frac{1}{2} K_{1}$ is the moment per unit width acting in the bilayer and is independent of $\epsilon_{o}, K_{2}$ is the in-plane force per unit width acting in the bilayer and depends on $\epsilon_{o}, K_{3}$ measures the asymmetry of the bilayer resulting from different Young's moduli and thicknesses of the two layers, $K_{4}$ is the bilayer bending stiffness per unit width, and $4 K_{5}$ is the bilayer longitudinal stiffness per unit width.

For free-free beams, the boundary conditions as derived from the PMPE are

$$
\begin{aligned}
& K_{1}+K_{3} v_{x}^{2}+2 K_{4} v_{x x}=0, \quad x=-L, L, \\
& K_{2} v_{x}-K_{4} v_{x x x}+2 K_{5} v_{x}^{2}=0, \quad x=-L, L .
\end{aligned}
$$

If Eq. (25) is used, the $K_{2} v_{x x}$ term in governing Eq. (29) and $K_{2} v_{x}$ term in the boundary conditions disappear. The difference between Eqs. (25) and (27) leads to the different governing equations and the boundary conditions, and thus will significantly affect the curvature computation. We linearize Eqs. (29) and (31) for a free-free beam in the small deflection case,

$$
K_{4} v_{x x x x}-K_{2} v_{x x}=0,
$$

and

$$
\begin{aligned}
& K_{1}+2 K_{4} v_{x x}=0, \quad x=-L, L, \\
& K_{2} v_{x}-K_{4} v_{x x x}=0, \quad x=-L, L .
\end{aligned}
$$

The solution of Eq. (32) with its boundary conditions of Eq. (33) is

$$
v(x)=\left\{\begin{array}{l}
-\frac{K_{1}}{2 K_{4} \cosh (\beta L) \beta^{2}} \cosh (\beta x)+C_{3} x+C_{4}, \quad \epsilon_{o}>0 \\
\frac{K_{1}}{2 K_{4} \cos (\beta L) \beta^{2}} \cos (\beta x)+C_{3} x+C_{4}, \quad \epsilon_{o}<0 \\
-\frac{K_{1}}{4 K_{4}} x^{2}+C_{3} x+C_{4}, \quad \epsilon_{o}=0,
\end{array}\right.
$$

where $\beta$ is defined as follows:

$$
\beta=\sqrt{\left|\frac{K_{2}}{K_{4}}\right|} .
$$

In Eq. (34), $C_{3}$ and $C_{4}$ are the constants to be determined. For a free-free beam, the boundary condition $K_{2} v_{x}-K_{4} v_{x x x}$ $=0$ at $x=-L$ and $L$ is automatically satisfied; therefore, $C_{3}$ and $C_{4}$ cannot be determined. However, because $C_{3} x+C_{4}$ is a rigid body displacement (rotation and translation), $C_{3} x+C_{4}$ 
makes no contribution to the system's potential energy $V$ and thus has no influence on the governing equation or the boundary conditions. From Eq. (34), the curvature then becomes

$$
\kappa_{L}(x)=v_{x x}=\left\{\begin{array}{l}
-\frac{K_{1}}{2 K_{4} \cosh (\beta L)} \cosh (\beta x), \quad \epsilon_{o}>0 \\
-\frac{K_{1}}{2 K_{4} \cos (\beta L)} \cos (\beta x), \quad \epsilon_{o}<0 \\
-\frac{K_{1}}{2 K_{4}}, \quad \epsilon_{o}=0 .
\end{array}\right.
$$

With $K_{i}$ being as defined in Eq. (30), it is straightforward to demonstrate that

$$
\kappa_{F}=\kappa_{T}=-\frac{K_{1}}{2 K_{4}} .
$$

It follows that only if $\epsilon_{o}=0$ (no in-plane force), the bilayer does have a uniform curvature. In addition, the structural dimension $2 L$ has no influence on the curvature. For either $\epsilon_{o}>0$ (tensile in-plane force) or $\epsilon_{o}<0$ (compressive in-plane force), the curvature of the bilayer varies with position. Only at the two free ends $(x=-L$ and $L), \kappa_{L}$ is equal to $\kappa_{F}$. At the center $(x=0), \kappa_{L}$ differs most from $\kappa_{F}$. For the tensile case $\left(\epsilon_{o}>0\right), \kappa_{L}(0)=-K_{1} /\left[2 K_{4} \cosh (\beta L)\right]=\kappa_{F} / \cosh (\beta L)$. For the compressive case $\left(\epsilon_{o}<0\right), \quad \kappa_{L}(0)=-K_{1} /\left[2 K_{4} \cos (\beta L)\right]$ $=\kappa_{F} / \cos (\beta L)$. Consider now the parameter $\beta L$.

$$
\beta L=\sqrt{\left|\frac{K_{2}}{K_{4}}\right|} L \propto \frac{\sqrt{\left|\epsilon_{o}\right|} L}{h_{f}+h_{s}} .
$$

Clearly, $L$ appears as an argument in $\kappa_{L}$ when $\epsilon_{o} \neq 0$ and the parameter $\sqrt{\left|\epsilon_{o}\right|} L /\left(h_{f}+h_{s}\right)$ qualitatively determines how the curvature varies along the beam span.

For a beam with two ends simply supported (ss-ss), we have the following boundary conditions:

$$
\begin{aligned}
& K_{1}+2 K_{4} v_{x x}=0, \quad x=-L, L, \\
& v=0, \quad x=-L, L,
\end{aligned}
$$

and the solution of $v(x)$ is

$$
v(x)=\left\{\begin{array}{l}
-\frac{K_{1}}{2 K_{4} \cosh (\beta L) \beta^{2}}[\cosh (\beta x)-\cosh (\beta L)], \quad \epsilon_{o}>0 \\
\frac{K_{1}}{2 K_{4} \cos (\beta L) \beta^{2}}[\cos (\beta x)-\cos (\beta L)], \quad \epsilon_{o}<0 \\
-\frac{K_{1}}{4 K_{4}}\left(x^{2}-L^{2}\right), \quad \epsilon_{o}=0 .
\end{array}\right.
$$

The curvature of a ss-ss beam has the same expression as given in Eq. (36) for a free-free beam. So all the discussion on the curvature of a free-free beam is the same for ss-ss beam.
Both free-free and ss-ss beams have symmetric boundary conditions. We now examine the cantilever beam which is asymmetric. For cantilever beams, the boundary conditions are

$$
\begin{aligned}
& v=0, \quad v_{x}=0, \quad x=-L, \\
& K_{1}+2 K_{4} v_{x x}=0, \quad K_{2} v_{x}-K_{4} v_{x x x}=0, \quad x=L .
\end{aligned}
$$

The deflection solution of the cantilever beam is therefore

$$
v(x)=\left\{\begin{array}{l}
-\frac{K_{1}}{2 K_{4} \cosh (2 \beta L) \beta^{2}}[\cosh (\beta x+\beta L)-1], \quad \epsilon_{o}>0 \\
\left.\frac{K_{1}}{2 K_{4} \cos (2 \beta L) \beta^{2}}[\cos (\beta x+\beta L)-1)\right], \quad \epsilon_{o}<0 \\
-\frac{K_{1}}{4 K_{4}}\left(x^{2}+2 L x-3 L^{2}\right), \quad \epsilon_{o}=0,
\end{array}\right.
$$

and the curvature is

$$
\kappa_{L}(x)=v_{x x}=\left\{\begin{array}{l}
-\frac{K_{1}}{2 K_{4} \cosh (2 \beta L)} \cosh (\beta x+\beta L), \quad \epsilon_{o}>0 \\
-\frac{K_{1}}{2 K_{4} \cos (2 \beta L)} \cos (\beta x+\beta L), \quad \epsilon_{o}<0 \\
-\frac{K_{1}}{2 K_{4}}, \quad \epsilon_{o}=0 .
\end{array}\right.
$$

Evidently, cantilever beams also have a uniform curvature only if $\epsilon_{o}=0$. Similar conclusions were reached by Zhang et al. $^{32}$

\section{RESULTS AND DISCUSSION}

Consider the case of a germanium film and a silicon substrate, and assume a beam structure. We have $M_{f}=E_{f}$ $=105.08 \mathrm{GPa}$ and $M_{s}=E_{s}=150 \mathrm{GPa}, a_{f}=0.56574 \mathrm{~nm}$ and $a_{s}=0.54306 \mathrm{~nm}$, and $h_{f}$ and $h_{s}$ are fixed as $h_{f}=0.1 \mu \mathrm{m}$ and $h_{s}=1 \mu \mathrm{m}$. The curvature $\kappa_{F}\left(M_{f} / M_{s}, h_{f} / h_{s}\right)$ as given in Eq. (7) clearly indicates the effect of $M_{f} / M_{s}$ and $h_{f} / h_{s}$ on the curvature. For our purposes, we only vary $L$ and $\epsilon_{o}$ to show their effects on the curvature $\kappa_{L}$ and introduce the following dimensionless ratios:

$$
\bar{\kappa}_{F}=\bar{\kappa}_{T}=\frac{\kappa_{F}}{\kappa_{\mathrm{St}}} \text { and } \bar{\kappa}_{L}=\frac{\kappa_{L}}{\kappa_{S t}} .
$$

Figure 2 shows the $\bar{\kappa}_{L}$ variations along the free-free or ss-ss beam span with different $\epsilon_{o}$ 's and $L=5.5 \mu \mathrm{m}\left[\left(h_{f}+h_{s}\right) / L\right.$ $\left.=\frac{1}{5}\right]$. The contribution of the film thickness to the curvature is evaluated by means of Eq. (7), which yields $\bar{\kappa}_{F}$ $=0.80581$ thus indicating an error of about $20 \%$ when compared with Stoney's formula. For the nonzero in-plane force case $\left(\epsilon_{o} \neq 0\right)$, we have $\bar{\kappa}_{L}=\bar{\kappa}_{F}$ only at the two ends. For the tensile in-plane force case $\left(\epsilon_{o}>0\right), \bar{\kappa}_{L}$ varies according to $\left[\bar{\kappa}_{F} / \cosh (\beta L)\right] \cosh (\beta x)$. At $L=0, \bar{\kappa}_{L}$ has a minimum value of $0.60784\left(\epsilon_{o}=0.2 \%\right)$ and $0.695422\left(\epsilon_{o}=0.1 \%\right)$. For the compressive in-plane force case $\left(\epsilon_{o}<0\right), \bar{\kappa}_{L}$ varies according to $\left[\bar{\kappa}_{F} / \cos (\beta L)\right] \cos (\beta x)$. At $L=0, \bar{\kappa}_{L}$ has a maximum value 


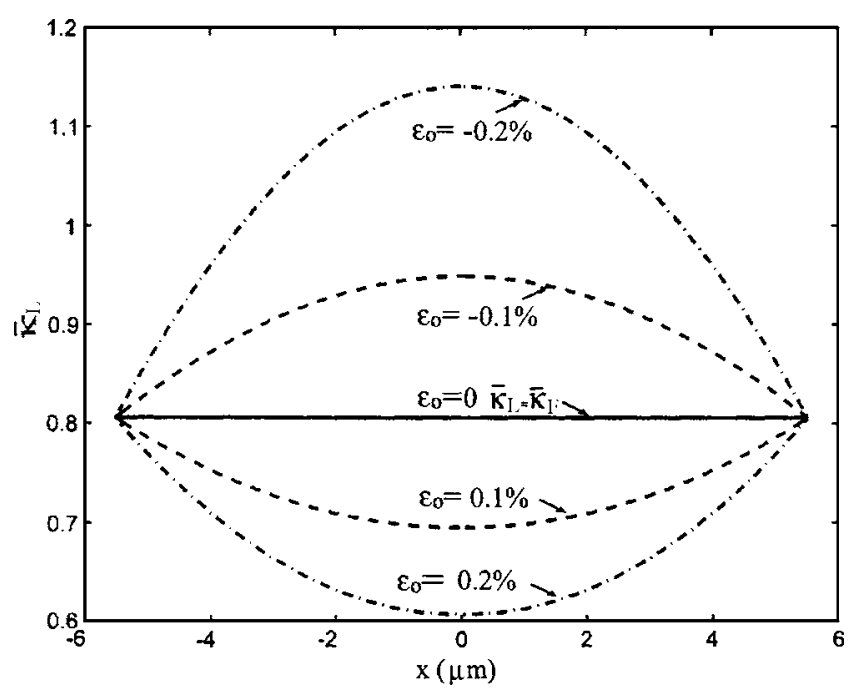

FIG. 2. Variation of the ratio $\bar{\kappa}_{L}$ along the free-free beam span for $h_{f}$ $+h_{s} / L=\frac{1}{5}$. Strains $\epsilon_{o}$ 's are set equal to $-0.2 \%,-0.1 \%, 0,0.1 \%$, and $0.2 \%$.

of $1.141\left(\epsilon_{o}=-0.2 \%\right)$ and $0.94885\left(\epsilon_{o}=-0.1 \%\right)$. Figure 3 shows the $\bar{\kappa}_{L}$ along the free-free/ss-ss beam span for different $\epsilon_{o}$ 's and $L=7.7 \mu \mathrm{m}\left[\left(h_{f}+h_{s}\right) / L=\frac{1}{7}\right]$. As $L$ increases $\left(h_{f}\right.$ and $h_{s}$ are fixed), the curvature becomes more and more sensitive to the in-plane forces. At $L=0, \bar{\kappa}_{L}$ with a tensile in-plane force now has the minimum values of $0.48245\left(\epsilon_{o}=0.2 \%\right)$ and $0.61099\left(\epsilon_{o}=0.1 \%\right)$. At $L=0, \bar{\kappa}_{L}$ with a compressive in-plane load now has the maximum values of $1.78102\left(\epsilon_{o}\right.$ $=-0.2 \%)$ and $1.13206\left(\epsilon_{o}=-0.1 \%\right)$.

Figure 4 shows the $\bar{\kappa}_{L}$ variation along the cantilever beam for different $\epsilon_{o}$ 's and $L=5.5 \mu \mathrm{m}\left[\left(h_{f}+h_{s}\right) / L=\frac{1}{5}\right]$. Only when $\epsilon_{o}=0$, the state of a uniform curvature is reached. The same $\bar{\kappa}_{L}=\bar{\kappa}_{F}=0.80581$ applies to the entire beam span. It is shown that for $\epsilon_{o}>0, \quad \bar{\kappa}_{L}$ varies according to $\left[\kappa_{F} / \cosh (2 \beta L)\right] \cosh (\beta x+\beta L)$. Its minimum is reached at the fixed end of $x=-L$ and $\bar{\kappa}_{L}=0.32041\left(\epsilon_{o}=0.2 \%\right)$ and $\bar{\kappa}_{L}$ $=0.54991\left(\epsilon_{o}=0.07 \%\right)$. For $\epsilon_{o}<0, \bar{\kappa}_{L}$ varies according to $\left[\kappa_{F} / \cos (2 \beta L)\right] \cos (\beta x+\beta L)$. Its maximum is reached at the fixed end of $x=-L$ and $\bar{\kappa}_{L}=1.47996\left(\epsilon_{o}=-0.08 \%\right)$ and $\bar{\kappa}_{L}$

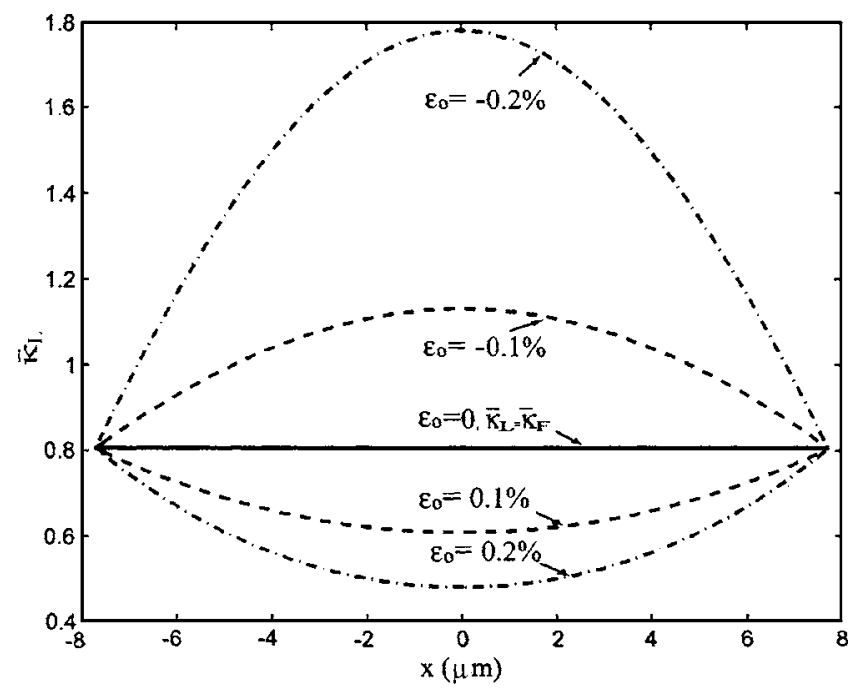

FIG. 3. Variation of the ratio $\bar{\kappa}_{L}$ along the free-free beam span for $h_{f}$ $+h_{s} / L=\frac{1}{7}$. Strains $\epsilon_{o}$ 's are set equal to $-0.2 \%,-0.1 \%, 0,0.1 \%$, and $0.2 \%$.

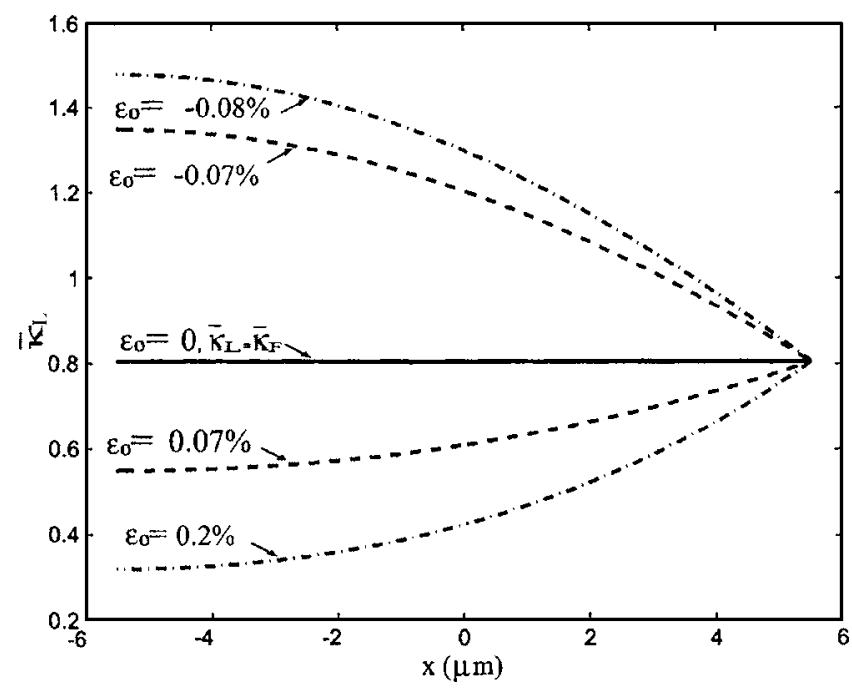

FIG. 4. Variation of the ratio $\bar{\kappa}_{L}$ along the cantilever beam span for $h_{f}$ $+h_{s} / L=\frac{1}{5}$. Strains $\epsilon_{o}$ 's are set equal to $-0.08 \%,-0.07 \%, 0,0.07 \%$, and $0.2 \%$.

$=1.3493\left(\epsilon_{o}=-0.07 \%\right)$. Figure 5 shows the $\bar{\kappa}_{L}$ variation along the cantilever beam with different $\epsilon_{o}$ 's and $L$ $=7.7 \mu \mathrm{m}\left[\left(h_{f}+h_{s}\right) / L=\frac{1}{7}\right]$. For the tensile case, at $x=-L, \bar{\kappa}_{L}$ $=0.17596\left(\epsilon_{o}=0.2 \%\right)$ and $0.40777\left(\epsilon_{o}=0.07 \%\right)$. Compared to Fig. 4 , the variation of $\bar{\kappa}_{L}$ for nonzero $\epsilon_{o}$ 's in Fig. 5 increases dramatically.

\section{CONCLUSION}

Our major conclusions are as follows. (i) In the absence of in-plane force, the assumption of a uniform curvature is always valid for small deflections; the $\kappa_{F}$ or $\kappa_{T}$ expressions for curvature are accurate. The curvature is determined only by layers' effective Young's moduli and the thickness ratio; furthermore, $\kappa_{F}=\kappa_{\mathrm{St}}$ for a very thin film. (ii) If there are in-plane forces, the kinematic assumption of a uniform curvature is not valid. Boundary conditions, structure dimensions, and in-plane force will all affect the variation of the

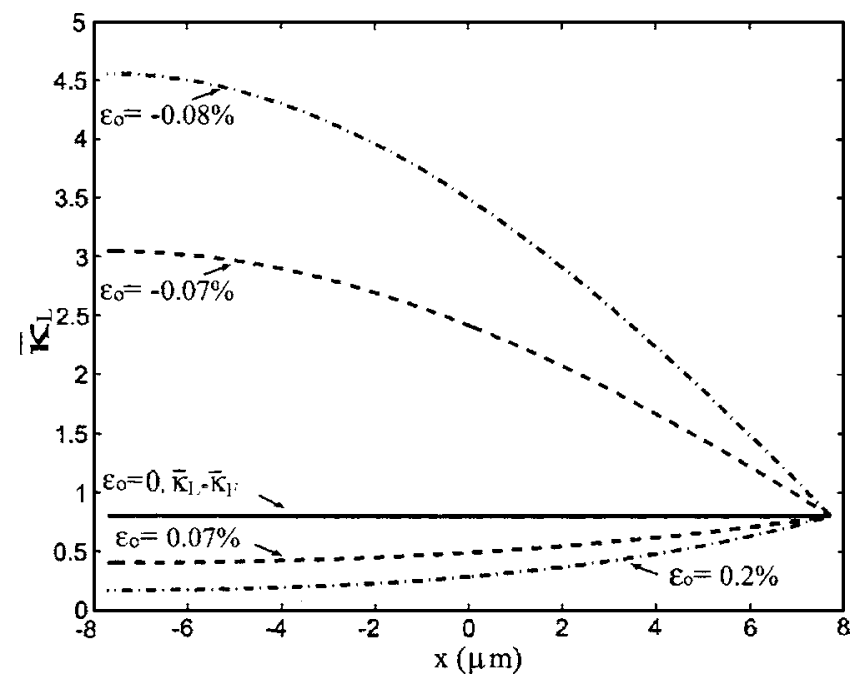

FIG. 5. Variation of the ratio $\bar{\kappa}_{L}$ along the cantilever beam span for $h_{f}$ $+h_{s} / L=\frac{1}{7}$. Strains $\epsilon_{o}$ 's are set equal to $-0.08 \%,-0.07 \%, 0,0.07 \%$, and $0.2 \%$. 
curvature along the span. (iii) The kinematic assumption as expressed by Eq. (25) does not include any in-plane force effects in either the governing equation or the boundary conditions. Only when the contribution of the nonlinear term $\left\{\frac{1}{2}[d v(x) / d x]^{2}\right\}$ due to the neutral plane stretching is included in Eq. (27), the in-plane force effects can be assessed. The structure dimensions (size effect) and boundary conditions can then affect both the deflection and the curvature.

Besides the six conditions concerning the applicability range of Stoney's formula, ${ }^{9}$ we introduce two more. (i) The interface is "ideal" in the sense of no interfacial slip. (ii) There is no in-plane force. These two conditions also apply to the modified Stoney's formula as $\kappa_{F}$.

\section{ACKNOWLEDGMENTS}

One of the authors (Y.Z.) is thankful to the financial support from both the National Natural Science Foundation of China (NSFC) (Grant No. 10502050) and the Scientific Research Foundation for the Returned Overseas Chinese Scholars, State Education Ministry. Another author (Y.P.Z.) is supported by the Distinguished Young Scholar Fund of the National Natural Science Foundation of China (NSFC) (Grant No. 10225209), a key project from the Chinese Academy of Sciences (Grant No. KJCX-SW-L2), and a NSFC project (Grant No. 90305020).

${ }^{1}$ G. G. Stoney, Proc. R. Soc. London 82, 172 (1909).

${ }^{2}$ K. Röll, J. Appl. Phys. 47, 3224 (1976).

${ }^{3}$ Z. Feng and H. Liu, J. Appl. Phys. 54, 83 (1982).

${ }^{4}$ P. H. Townsend, D. M. Barnett, and T. A. Brunner, J. Appl. Phys. 62 , 4438 (1987).

${ }^{5}$ H. C. Liu and S. P. Murarka, J. Appl. Phys. 72, 3458 (1992).

${ }^{6}$ M. Finot and S. Suresh, J. Mech. Phys. Solids 44, 683 (1996).
${ }^{7}$ L. B. Freund and S. Suresh, Thin Film Materials: Stress, Defect Formation and Surface Evolution (Cambridge University Press, Cambridge, UK, 2003), Chaps. 2 and 6.

${ }^{8}$ L. B. Freund, J. Mech. Phys. Solids 44, 723 (1996).

${ }^{9}$ L. B. Freund, J. A. Floro, and E. Chason, Appl. Phys. Lett. 74, 1987 (1999).

${ }^{10}$ C. A. Klein and R. P. Miller, J. Appl. Phys. 87, 2265 (2000).

${ }^{11}$ C. H. Hsueh, J. Appl. Phys. 91, 9652 (2002).

${ }^{12}$ C. H. Hsueh and S. Lee, J. Appl. Mech. 70, 151 (2003).

${ }^{13}$ Y. Y. Hu and W. M. Huang, J. Appl. Phys. 96, 4154 (2004).

${ }^{14}$ C. A. Klein, J. Appl. Phys. 88, 5487 (2000).

${ }^{15}$ Q. H. Fan, A. Fernandes, E. Pereira, and J. Grácio, J. Appl. Phys. 84, 3155 (1998).

${ }^{16}$ F. Q. Yang and J. C. M. Li, J. Appl. Phys. 93, 9304 (2003).

${ }^{17}$ S. P. Timoshenko, J. Opt. Soc. Am. 11, 233 (1925).

${ }^{18} \mathrm{~S}$. P. Timoshenko and S. Woinowsky-Krieger, Theory of Plates and Shells, 2nd ed. (McGraw-Hill, New York, 1959), p. 5.

${ }^{19}$ C. Q. Ru, J. Electron. Packag. 124, 141 (2002).

${ }^{20}$ C. E. Murray and I. C. Noyan, Philos. Mag. A 82, 3087 (2002).

${ }^{21}$ Y. Okuno, K. Uomi, M. Aoki, T. Taniwatari, M. Suzuki, and M. Kondow, Appl. Phys. Lett. 66, 451 (1995).

${ }^{22}$ F. Shi, S. MacLaren, C. Xu, K. Y. Cheng, and K. C. Hsieh, J. Appl. Phys. 93, 5750 (2003).

${ }^{23}$ F. Shi, K. Chang, K. C. Hsieh, L. Guido, and B. Hoke, J. Appl. Phys. 95, 909 (2004).

${ }^{24}$ Y. Benveniste, Mech. Mater. 3, 349 (1984)

${ }^{25}$ I. C. Noyan, C. E. Murray, J. S. Chey, and C. C. Goldsmith, Appl. Phys. Lett. 85, 724 (2004).

${ }^{26}$ W. T. Chen and C. W. Nelson, IBM J. Res. Dev. 23, 179 (1979).

${ }^{27}$ E. Suhir, J. Appl. Mech. 53, 657 (1986).

${ }^{28}$ E. Suhir, J. Appl. Mech. 56, 595 (1989).

${ }^{29}$ Freund's energy analysis as given in the paper is for circular plate structures, and Timoshenko's analysis is for rectangular beams or plates. But applying Freund's energy analysis to the rectangular beams or plates does not modifies the form of Eq. (7); only the effective moduli $M_{f}$ and $M_{s}$ are different.

${ }^{30}$ F. P. Beer and E. R. Johnson, Jr., Mechanics of Material (McGraw-Hill, New York, 1981), pp. 168-170.

${ }^{31}$ A. L. Thurman and C. D. Mote, J. Appl. Mech. 36, 83 (1969).

${ }^{32}$ Y. Zhang, Q. Ren, and Y. P. Zhao, J. Phys. D 37, 2140 (2004). 